\title{
On a method of acceleration of convergence
}

By

\author{
Sin Hitotumatu*
}

\section{§. Introduction}

In a recent paper [4], the author discussed the numerical computation of incomplete or complete gamma function through power series and continued fraction.

When we use the continued fraction, the convergence of the successive approximative values is rather slow, so that a certain accelaration is desirable. In the present paper, the author discusses a method of acceleration, which is a slight modification of Aitken's formula and of quite general character.

The author is indepted to $\mathrm{CDC} 6600$ in the computing center at Brookhaven National Laboratory, ${ }^{1)}$ for various numerical experiments.

\section{§1. The original problem}

The original formura for the incomplete gamma function is as follows:

$$
\begin{aligned}
& \int_{0}^{x} e^{-t} t^{\nu-1} d t=x^{\nu} e^{-x} \sum_{n=1}^{N} \frac{(1-\nu)(2-\nu) \cdots(n-1-\nu)}{(-x)^{n}} \\
& \quad+x^{\nu}(1-\nu)(2-\nu) \cdots(N-\nu) \sum_{n=0}^{\infty} \frac{(-x)^{n-N}}{n !(n+\nu-N)} \\
& =x^{\nu} e^{-x} \sum_{n=1}^{N} \frac{(1-\nu)(2-\nu) \cdots(n-1-\nu)}{(-x)^{n}}+\Gamma(\nu)
\end{aligned}
$$

Received October 31, 1966.

Communicated by M. Hukuhara.

* Department of Mathematics, St. Paul's University (Tokyo) and Applied Mathematics Department, Brookhaven National Laboratory.

1) Problem AM041. 


$$
\begin{aligned}
& -\frac{x^{\nu} e^{-x}}{(-x)^{N}}(1-\nu)(2-\nu) \cdots(N-\nu)\left[\frac{1 \mid}{\mid x}+\frac{N-\nu+1 \mid}{1}\right. \\
& \left.+\frac{1 \mid}{\mid x}+\frac{N-\nu+2 \mid}{\mid}+\frac{2 \mid}{\mid x}+\cdots\right] .
\end{aligned}
$$

The first term is the asymptotic expansion of the incomplete gamma function and the final term means a continued fraction. Its successive approximative values $p_{n} / q_{n}$ are given by the following recurrence formula

$$
\begin{gathered}
p_{-1}=0, p_{0}=1, q_{-1}=1, q_{0}=x \\
q_{2 n+1}=q_{2 n}+(N+n+1-\nu) q_{2 n-1}, q_{2 n+2}=x q_{2 n+1}+(n+1) q_{2 n} \\
(n=0,1,2, \cdots) .
\end{gathered}
$$

The same recurrence formulas hold for $p_{n}$ 's, too.

The formulas are true for arbitrary complex values of $x$ and $\nu$, except when $x$ is real negative, and in addition, for the first power series some modifications are necessary when $\nu$ is a non-positive integer. $N$ may be an arbitrary non-negative integer, but in practice, $N$ is chosen such that $|N-\nu|-|x|$ becomes as small as possible. By a recurrence formula of the incomplete gamma function, we may restrict our attention to $0 \leqq \operatorname{Re} \nu<1$, without loss of generality.

Now, the convergence of the sequence $p_{n} / q_{n}$ is rather slow. For example, we need 27 repetitions when $x=1.0, \nu=0$ for the accuracy $10^{-8}$, and 54 repetitions when $x=1.0, \nu=0.5$ for the accuracy $10^{-12}$. We remark that throughout the paper, we count the whole application of (2) as one repetition, so that $a_{n}=p_{2 n} / q_{2 n}$ is the $n$-th approximate value. When $x$ is real positive and $\nu \geqq 0$, in which case we are mainly interested, the sequence $p_{n} / q_{n}$ itself converges oscillatorily, and the partial sequences $p_{2 n} / q_{2 n}$ and $p_{2 n-1} / q_{2 n-1}$ converge monotonously (cf. [5], [7]). This suggests that the mean value

$$
\frac{1}{2}\left(\frac{p_{2 n-1}}{q_{2 n-1}}+\frac{p_{2 n}}{q_{2 n}}\right)
$$

will give a simple acceleration. (3) is effective also for complex argument $x$, but we need still an alternative acceleration. 
It is known (cf. [3] $)^{2)}$ that the absolute value of the truncation error of $p_{n} / q_{n}$ is asymptotically of the form

$$
A^{*} \varepsilon^{* \sqrt{n}}, 0<\varepsilon^{*}<1, A^{*}>0
$$

where $A^{*}$ and $\varepsilon^{*}$ are constants depending upon $x, \nu$ and $N$. As is seen from this, the convergence is rapid at first and becomes slower and slower successively; but when $|x|$ is large enough, $\varepsilon^{*}$ being small, we may fortunately stop the repetitions before the convergence becomes very slow.

From the remark given above, the principal error term for $a_{n}$ $==p_{2 n} / q_{2 n}$ is of the form

$$
A \varepsilon^{\sqrt{n}}, \varepsilon=\varepsilon^{* \sqrt{2}}, 0<\varepsilon<1 ; A= \pm A^{*}
$$

when $x>0, \nu \geqq 0$. Though we have the expressions of the constants $A$ and $\varepsilon$ theoretically, we shall mainly use the fact that the main error term has a form like (4) for some constants $A, \varepsilon$, in the following.

\section{§. Modified Aitken's method}

For a while, let us assume that the error of $a_{n}=p_{2 n} / q_{2 n}$ itself is just (4). Then three consecutive approximative values are

$$
\left\{\begin{aligned}
a_{n-1} & =V+A \varepsilon^{\sqrt{n-1}}, \\
a_{n} & =V+A \varepsilon^{\sqrt{n}}, \\
a_{n+1} & =V+A \varepsilon^{\sqrt{n+1}},
\end{aligned}\right.
$$

respectively, where $V$ is the true value.

We put $\sqrt{n-1}=k, \sqrt{n}=l, \sqrt{n+1}=m$ for the sake of simplicity. Remark that the second difference

$$
\delta=(l-k)-(m-l)
$$

is much smaller than the values of $k, l, m$ and even if compared with the first differences $l-k, m-l$. In fact, we have, as the first approximation, the following formulas

2) In paper [3], only the case $\nu=1 / 2$ is discussed, but similar theorems hold also for $0 \leqq \nu<1$. 
( 7 )

$$
\left\{\begin{array}{l}
\delta \doteqdot \frac{\sqrt{n}}{4 n^{2}-(5 / 4)} \quad \text { or roughly } \quad \frac{\sqrt{n}}{4 n^{2}-1}, \\
l-k \doteqdot \sqrt{n} /(2 n-1 / 2), \\
m-l \doteqdot \sqrt{n} /(2 n+1 / 2) .
\end{array}\right.
$$

Now, we begin with a more general formula:

$$
\left\{\begin{aligned}
a_{n-1} & =V+A \varepsilon^{k}, \\
a_{n} & =V+A \varepsilon^{l}, \\
a_{n+1} & =V+A \varepsilon^{m},
\end{aligned}\right.
$$

instead of (5), and assume that (6) is small compared with $l-k$, $m-l$. Then it is easy to see that

$$
\begin{aligned}
& a_{n+1}-a_{n}=A \varepsilon^{l}\left(\varepsilon^{m-l}-1\right)=A \varepsilon^{m}\left(1-\varepsilon^{-(n-l)}\right), \\
& a_{n}-a_{n-1}=A \varepsilon^{k}\left(\varepsilon^{l-k}-1\right)
\end{aligned}
$$

and then

$$
\begin{aligned}
\rho & =\frac{a_{n+1}-a_{n}}{a_{n}-a_{n-1}}=\varepsilon^{l-k} \frac{\varepsilon^{l-k-\delta}-1}{\varepsilon^{l-k}-1} \\
& =\varepsilon^{l-k-\delta}\left(1-\frac{1-\varepsilon^{\delta}}{1-\varepsilon^{l-k}}\right)=\varepsilon^{m-l} \cdot \Delta,
\end{aligned}
$$

where we have put

$$
\Delta=1-\frac{1-\varepsilon^{\delta}}{1-\varepsilon^{l-k}} .
$$

We call $\Delta$ the modification factor.

Though $\Delta$ is a function of $\varepsilon$, we may estimate it in various ways as in the next paragraph, hence we proceed henceforth as if $\Delta$ is a known quantity. Then we have

$$
A \varepsilon^{m}=\frac{a_{n+1}-a_{n}}{1-\varepsilon^{-(m-l)}}=\frac{a_{n+1}-a_{n}}{1-\Delta / \rho}=\frac{\left(a_{n+1}-a_{n}\right)^{2}}{\left(a_{n+1}-a_{n}\right)-\Delta\left(a_{n}-a_{n-1}\right)},
$$

and finally

$$
V=a_{n+1}-\frac{\left(a_{n+1}-a_{n}\right)^{2}}{\left(a_{n+1}-a_{n}\right)-\Delta\left(a_{n}-a_{n-1}\right)} .
$$

If $\delta$ is precisely 0 , we have $\Delta=1$ and the formula (11) is nothing but the so-called Aitken's acceleration formula or the Aitken's $\delta^{2}$ algorithm ([1]; see also [2] Chap. 4, or [6]). Hence (11) may be 
called a modified Aitken's method.

In the practical use, it will be more convenient to write the denominator of (11) as

$$
\left[\left(a_{n+1}-a_{n}\right)-\left(a_{n}-a_{n-1}\right)\right]+\gamma\left(a_{n}-a_{n-1}\right)
$$

where we have put

$$
r=1-\Delta=\frac{1-\varepsilon^{\delta}}{1-\varepsilon^{l-k}} .
$$

The first term in (12) is the second difference of (8).

Now, in our original problem, the right hand side of (11) is not the precise value $V$, because (4) itself is not the actual error, but its main term. Nevertheless, we may expect that the value defined by (11) much improves the approximation, if we choose $\Delta$ properly.

\section{§3. The determination of the modification factor $\Delta$}

In the original problem in $\S 1$, we may have a possibility to choose $\Delta=1$, taking $a_{1}, a_{4}, a_{9}$ or $a_{4}, a_{9}, a_{16}$ instead of three consecutive approximations (5). Or, simply neglecting $\delta$, we may assume $\Delta=1$. However, these procedures do not give satisfactory results in practice, and we had better to look for the factor $\Delta$.

i) As for (5), one of the selection of $\Delta$ is the following:

$$
\Delta \doteqdot \Delta_{1}=\frac{16 n^{2}-8 n-3}{16 n^{2}-5}, \quad \gamma \doteqdot \gamma_{1}=\frac{2(4 n-1)}{16 n^{2}-5},
$$

or, neglecting smaller terms we may put

$$
\Delta \doteqdot \Delta_{1}^{\prime}=\frac{4 n-1}{4 n+1}, \quad \gamma \doteqdot \gamma_{1}^{\prime}=\frac{2}{4 n+1} .
$$

(13) comes from the following considerations. Applying the approximation (7) we have

$$
\gamma=1-\Delta \doteqdot \frac{1-\varepsilon^{\sqrt{n} /(4 n 2-5 / 4)}}{\varepsilon^{\sqrt{n} \mid(2 n-1 / 2)}}=\frac{1-\alpha}{1-\alpha^{\lambda}},
$$

where we put

$$
\alpha=\varepsilon^{\sqrt{n} /\left(4 n^{2}-5 / 4\right)}, \quad \lambda=\left(4 n^{2}-5 / 4\right) /(2 n-1 / 2) \doteqdot 2 n+1 / 2 .
$$

In general, $\varepsilon$ may be assumed not so small; otherwise we need no 
particular acceleration. Hence we may assume that $\alpha$ is fairly close to 1. (13) is nothing but the limit of the right hand side of (14) when $\alpha$ tends to 1 .

Many examples show that (13) gives much better results compared with the choice $\Delta=1$. However, in the original problem, some ex periments show that it will be better to take

$$
\Delta \doteqdot \Delta_{1}^{*}=\frac{2 n-2}{2 n-1}, \quad \gamma \doteqdot \gamma_{1}^{*}=\frac{1}{2 n-1}
$$

instead of (13).

ii) Another choice of $\Delta$ is the following:

$$
\Delta \doteqdot \Delta_{2}=\frac{\beta\left(1-\beta^{2 n-1 / 2}\right)}{1-\beta^{2 n+1 / 2}}, \quad \gamma \doteqdot \gamma_{2}=\frac{1-\beta}{1-\beta^{2 n+1 / 2}},
$$

where we put

$$
\beta=\left[\left(1+\frac{2}{4 n-1}\right) \rho\right]^{1 /(2 n-1 / 2)} .
$$

If $\beta=1, \gamma$ is replaced by $1 /(2 n+1 / 2)$, and then (17) reduces to $\left(13^{\prime}\right)$.

(17) comes from the following approximation of (14). From (9) we have

$$
\varepsilon^{m-l}=\rho / \Delta .
$$

Applying the approximations (7) and $\left(13^{\prime}\right)$, we may assume

$$
\varepsilon^{\sqrt{n} /(2 n+1 / 2)} \doteqdot \frac{4 n+1}{4 n-1} \rho
$$

which gives

$$
\alpha \doteqdot \varepsilon^{\sqrt{n} /\left(4 n^{2}-1 / 4\right)} \doteqdot\left[\left(1+\frac{2}{4 n-1}\right) \rho\right]^{1 /(2 n-1 / 2)} .
$$

iii) As will be shown below, there is a similar but alternative selection of $\beta$ in (17), say $\beta=\rho^{1 / 2 n}$, i. e.,

$$
\Delta \doteqdot \Delta_{2}^{\prime}=\rho^{1 / 2 n} \frac{1-\rho^{1-1 / 4 n}}{1-\rho^{1+1 / 4 n}}, \quad r \doteqdot \gamma_{2}^{\prime}=\frac{1-\rho^{1 / 2 n}}{1-\rho \rho^{1 / 4 n}} .
$$

Let us consider other choices of $\Delta$. In the original problem, approximative equations

$$
1-\frac{\rho}{\alpha^{2 n-1 / 2}} \doteqdot 1-\Delta \doteqdot \frac{1-\alpha}{1-\alpha^{2 n+1 / 2}}
$$


hold, where $\alpha$ is given by (15). (19) follows easily from (7), (9) and (14). If we equate both sides of (19), we have

$$
\rho\left(1-\alpha^{2 n+1 / 2}\right)=\alpha^{2 n+1 / 2}\left(1-\alpha^{2 n-1 / 2}\right) .
$$

Remembering that $\alpha$ is close to 1 , it is easy to see that (20) has a root $\alpha$ very near to $\rho^{1 / 2 n}$, which corresponds to the $\Delta$ for our present purpose. If we put $\alpha=\rho^{1 / 2 n}$ and insert it into (14), we have just (18) given above.

iv) In order to have a more precise approximation, we replace $\alpha^{2 n+1 / 2}$ in both sides of (20) by $\alpha^{2 n} \rho^{1 / 4 n}$, since $\alpha$ is close to $\rho^{1 / 2 n}$. After this replacement, (20) reduces to a quadratic equation if we put

say:

$$
\alpha^{2 n}=\rho+t
$$

We need the smaller root of (21), since (21) has two positive roots and $t$ must be very small. It is

$$
t=\frac{1}{2} \rho^{1 / 4 n}(\rho+1)-\rho-\frac{1}{2} \rho^{1 / 4 n}(1-\rho)\left[1-\frac{4 \rho\left(1-\rho^{1 / 2 n}\right)}{\rho^{1 / 2 n}(1-\rho)^{2}}\right]^{1 / 2} .
$$

Noting $0<\rho<1$ and $1-\rho^{1 / 2 n}$ being small, we expand the last term, and it is approximately

$$
\begin{aligned}
t \doteqdot \frac{1}{2} \rho^{1 / 4 n}(\rho+1)-\rho-\frac{1}{2} \rho^{1 / 4 n}(1-\rho)+\frac{\rho\left(1-\rho^{1 / 2 n}\right)}{\rho^{1 / 4 n}(1-\rho)} \\
=\frac{\rho\left(1-\rho^{1 / 4 n}\right)\left(1+\rho \rho^{1 / 4 n}\right)}{\rho^{1 / 4 n}(1-\rho)} .
\end{aligned}
$$

The root (22) gives us

$$
\alpha^{2 n} \doteqdot \frac{\rho^{1-1 / 4 n}\left(1-\rho^{1+1 / 2 n}\right)}{1-\rho}
$$

and hence

$$
\begin{aligned}
\Delta & \doteqdot \Delta_{3}=\frac{\rho}{\alpha^{2 n-1 / 2}}=\left(\frac{\rho}{\alpha^{2 n}}\right)^{1-1 / 4 n} \rho^{1 / 4,2} \\
& =\left(\frac{1-\rho}{1-\rho^{1+1 / 2 n}}\right)^{1-1 / 4 n} \rho^{1 / 2 n-1 /(4 n)^{2}} .
\end{aligned}
$$

We again mention that $\rho^{1 / 2 n}$ is close to 1 , and hence (23) may be 
replaced simply by

$$
\Delta_{4}=\rho^{1 / 2 n} \cdot \frac{1-\rho}{1-\rho^{1+1 / 2 n}}
$$

or, noting

$$
X^{1-1 / p} \doteqdot X+\frac{1}{p}(1-X)
$$

if $X$ is sufficiently near 1 , we may replace (23) by

$$
\Delta_{5}=\left[\left(1-\frac{1}{4 n}\right) \frac{1-\rho}{1-\rho \rho^{1 / 2 n}}+\frac{1}{4 n}\right] \rho^{1 / 2 n-1 /(4 n)^{2}} .
$$

v) So far, we have discussed various approximations of $\Delta$. In most cases, there are only little differences among the values (17), (18), (23), (24) and (25). For example, the difference between (18) and (24) is

$$
-\rho^{1+1 / 4 n} \frac{\left(1-\rho^{1 / 2 n}\right)\left(1-\rho^{1 / 4 n}\right)}{\left(1-\rho \rho^{1 / 2 n}\right)\left(1-\rho \rho^{1 / 4 n}\right)}
$$

whose absolute value is about $3.6 \times 10^{-3}$ when $\rho=0.3, n=5$. Later we give some examples of the various choices of the values of $\Delta$. Probably, $\Delta_{1}^{\prime}$ in $\left(13^{\prime}\right)$ or $\Delta_{2}^{\prime}$ in (18) will be the simplest and the most preferable approximation of $\Delta$ in the practical application.

\section{\$4. Four point acceleration}

The acceleration method discussed above uses three consecutive terms. There are other formulas using four consecutive terms.

In the case of linear convergence, i. e., if

$$
a_{n}=V+A \varepsilon^{n},
$$

there is an alternative acceleration method, say:

$$
V=a_{n+2}-\frac{\left(a_{n+2}-a_{n+1}\right)\left(a_{n+2}-a_{n}\right)}{\left(a_{n+2}-a_{n+1}\right)-\left(a_{n}-a_{n-1}\right)}=\frac{a_{n+2} a_{n-1}-a_{n} a_{n+1}}{a_{n+2}-a_{n+1}-a_{n}+a_{n-1}} .
$$

Under the assumption (5), (26) should be modified as follows:

$$
V=a_{n+2}-\frac{\left(a_{n+2}-a_{n+1}\right)\left(a_{n+2}-a_{n}\right)}{\left(a_{n+2}-a_{n+1}\right)-\Delta\left(a_{n}-a_{n-1}\right)},
$$

where 
(28)

$$
\Delta=1-\frac{1-\varepsilon^{-\sqrt{n+2}+\sqrt{n+1}+\sqrt{n}-\sqrt{n-1}}}{1-\varepsilon^{\sqrt{n}}-\sqrt{n-1}} .
$$

Since $\alpha=\varepsilon^{-\sqrt{n+2}+\sqrt{n+1}+\sqrt{n}-\sqrt{n-1}}$ is close to 1 , and

$$
\frac{\sqrt{n}-\sqrt{n-1}}{-\sqrt{n+2}+\sqrt{n+1}+\sqrt{n}-\sqrt{n-1}} \doteqdot n+1,
$$

we may use

$$
\Delta \doteqdot 1-\frac{1}{n+1}=\frac{n}{n+1}
$$

\begin{tabular}{|c|c|c|c|c|}
\hline & & Example 1 & Example 2 & \\
\hline \multicolumn{2}{|c|}{$\nu$} & 0 & 0 & 0.5 \\
\hline \multicolumn{2}{|c|}{$x$} & 2.0 & 3.0 & 1.0 \\
\hline \multicolumn{2}{|c|}{$N$} & 1 & 3 & 0 \\
\hline \multicolumn{2}{|c|}{$n$} & 5 & 3 & 4 \\
\hline \multicolumn{2}{|c|}{$a_{n-1}$} & 0.01878318876 & 0.001721034461 & -0.2783221014 \\
\hline \multicolumn{2}{|c|}{$a_{n}$} & 0.01877201332 & 0.001706356128 & -0.2786468689 \\
\hline \multicolumn{2}{|c|}{$a_{n+1}$} & 0.01876876153 & 0.001703968113 & -0.2787464214 \\
\hline \multicolumn{2}{|c|}{$\rho$} & 0.290976 & 0.162690 & 0.306535 \\
\hline \multicolumn{2}{|c|}{$\Delta_{1}$} & 0.903798 & 0.841727 & 0.880478 \\
\hline \multicolumn{2}{|c|}{$\Delta_{1}^{\prime}$} & 0.904762 & 0.846154 & 0.882353 \\
\hline \multicolumn{2}{|c|}{$\Delta_{1}^{*}$} & 0.888889 & 0.800000 & 0.857143 \\
\hline \multicolumn{2}{|c|}{$\Delta_{3}$} & 0.841209 & 0.697938 & 0.811618 \\
\hline \multicolumn{2}{|c|}{$\Delta_{2}^{\prime}$} & 0.840141 & 0.696402 & 0.808500 \\
\hline \multicolumn{2}{|c|}{$\Delta_{3}$} & 0.848213 & 0.714987 & 0.819785 \\
\hline \multicolumn{2}{|c|}{$\Delta_{4}$} & 0.843665 & 0.704315 & 0.813329 \\
\hline \multicolumn{2}{|c|}{$\Delta_{5}$} & 0.848257 & 0.715045 & 0.820079 \\
\hline \multirow{4}{*}{$V$} & $\Delta_{1}^{\prime}$ & $0.01876729966 \sim a_{9}$ & $0.001703399777 \sim a_{6}$ & -0.2787994150 \\
\hline & $\Delta_{1}^{*}$ & $0.01876718845 \sim a_{10}$ & $0.001703359580 \sim a_{7}$ & -0.2788018441 \\
\hline & $\Delta_{2}^{\prime}$ & $0.01876704142 \sim a_{9}^{\prime}$ & $0.001703241112 \sim a_{5}^{\prime}$ & -0.2788072089 \\
\hline & $\Delta_{5}$ & $0.01876706637 \sim a_{10}^{\prime}$ & $0.001703250871 \sim a_{5}^{\prime}$ & -0.2788058516 \\
\hline \multicolumn{2}{|c|}{$a_{n+2}$} & 0.01876771765 & 0.001703492462 & -0.2787813860 \\
\hline \multicolumn{2}{|c|}{$V^{*}$} & $0.01876717536 \sim a_{10}$ & $0.001703342621 \sim a_{9}$ & -0.2788023037 \\
\hline \multirow{2}{*}{\multicolumn{2}{|c|}{$\begin{array}{c}\text { true value } \\
\varepsilon \doteqdot\end{array}$}} & 0.0187671309 & 0.00170334287 & -0.2788055853 \\
\hline & & $7.0_{10}-3$ & $3.5_{10}-3$ & $\left(1.4_{10}-2\right)$ \\
\hline
\end{tabular}

Table 1

Table 2

$a_{n}=p_{2 n} / q_{2 n}, a_{n}^{\prime}=p_{2 n-1} / q_{2 n-1}$ in Table 1 ; and $a_{n}=$ the mean (3) in Table $2 . V$ is the value (11) using various approximation of $\Delta^{\prime}$ 's, and $V^{*}$ means the value (27), using $\Delta$ in (29). 
for the modification factor $\Delta$, similarly as (13). Of course, there are many other approximations of $\Delta$ in (28) as in the previous paragraph. But experiments show that (29) gives fairly good results.

\section{$\S 5 . \quad$ Numerical examples}

In Table 1, we give some examples of various approximations of $\Delta$ and the accelerated results for the term of continued fraction in (1). Table 2 is another example applying the formula (11) to the mean value (3). The results seem to be fairly good.

\section{REFERENCES}

[1] Aitken, A. C., On Bernoulli's numerical solution of algebraic equatoin, Proc. Roy. Soc, Edinburgh Sect. A 46 (1926), 289-305.

[2] Henrici, P., Elements of numerical analysis, Wiley, New York, 1964.

[3] , Error bounds for computations with continued fractions, Error in digital computations, Vol. 2, Wiley, New York (1965), 39-53.

[4] Hitotumatu, S., On the numerical computation of incomplete gamma function, Comment. Math. Univ. St. Paul. 15 (1967), 91-108.

[5] Perron, O., Die Lehrbuch von den Kettenbrüchen, Teubner, Leipzig, 1929.

[6] Traub, T. F., Iterative methods for the solution of equations, Prentice-Hall, New Jersey, 1964; especially Appendix D.

[7] Wall, H. S., Analytic theory of continued fraction, Van Nostrand, Princeton, 1948. 